\title{
Effects of the Dzyaloshinskii-Moriya interaction on low energy magnetic excitations in copper benzoate
}

\author{
J. Z. Zhao ${ }^{1}$, X. Q. Wang ${ }^{1,2,3}$, T. Xiang ${ }^{1,2}$, Z. B. $\mathrm{Su}^{1,2}$, and L. $\mathrm{Yu}^{1,2}$ \\ ${ }^{1}$ Institute of Theoretical Physics, CAS, Beijing 100080 China \\ ${ }^{2}$ The Interdisciplinary Center of Theoretical Studies, CAS, Beijing 100080, China and \\ ${ }^{3}$ Institute for Solid State Physics, University of Tokyo, Kashiwa, Chiba 277-8581, Japan
}

\begin{abstract}
We have investigated the physical effects of the Dzyaloshinskii-Moriya (DM) interaction in copper benzoate. In the low field limit, the spin gap is found to vary as $H^{2 / 3} \ln ^{1 / 6}\left(J / \mu_{B} H_{s}\right)\left(H_{s}\right.$ : an effective staggered field induced by the external field $H$ ) in agreement with the prediction of conformal field theory, while the staggered magnetization varies as $H^{1 / 3}$ and the $\ln ^{1 / 3}\left(J / \mu_{B} H_{s}\right)$ correction predicted by conformal field theory is not confirmed. The linear scaling behavior between the momentum shift and the magnetization is broken. We have determined the coupling constant of the DM interaction and have given a complete quantitative account for the field dependence of the spin gaps along all three principal axes, without resorting to additional interactions like interchain coupling. A crossover to strong applied field behavior is predicted for further experimental verification.
\end{abstract}

PACS numbers: 75.10.Jm, 75.50.Ee, 75.25.+z

Recently some novel magnetic properties were discovered in a variety of quasi-one dimensional materials, such as copper benzoate $\mathrm{Cu}\left(\mathrm{C}_{6} \mathrm{D}_{5} \mathrm{COO}\right)_{2} 3 \mathrm{D}_{2} \mathrm{O}$ 1, 2], $\mathrm{Yb}_{4} \mathrm{As}_{3}$ [3, 4, 5] and $\mathrm{BaCu}_{2} \mathrm{Si}_{2} \mathrm{O}_{7}[\underline{6}$. In these materials, the Dzyaloshinskii-Moriya (DM) interaction [7, 8, 9] plays an important role, especially in an applied magnetic field. This has stimulated extensive investigation on the physical properties of the DM interaction. However, this interaction is rather difficult to handle analytically, which has brought much uncertainty in the interpretation of experimental data and has limited our understanding of many interesting quantum phenomena of low-dimensional magnetic materials.

For copper benzoate, Dender et al [1, 2] found that the spin excitation gap shows a peculiar field dependence, $\Delta \sim H^{0.65}$, in low fields. On the contrary, excitations remain gapless in the $S=1 / 2$ Heisenberg model below a critical field. Oshikawa and Affleck (OA) suggested that this field dependence of the gap is due to a staggered magnetic field induced by the DM interaction in addition to the staggered g-factor in a uniform field $[9,10$. However, a satisfactory explanation for the field-dependence of the energy gaps in all three directions is still lacking 11, 12. It was argued that the inconsistency between the experimental data and theoretical results might be due to the neglect of the interchain coupling and/or anisotropic interaction terms in the low-field effective model used by Oshikawa and Affleck 9, 11]. We believe this issue can be clarified by a thorough study of the DM interaction and a direct comparison with experiments.

Copper benzoate is a quasi-1D spin- $1 / 2$ antiferromagnetic Heisenberg system. The chain direction is the caxis. It contains two types of alternating and slightly tilted $\mathrm{CuO}_{8}$ octahedra. This leads to two inequivalent $\mathrm{Cu}^{++}$ions and an alternating DM coupling [13. In an applied field, copper benzoate can be modeled by the following Hamiltonian,

$$
\begin{aligned}
\hat{H}=\sum_{i} & \left(J \hat{\mathbf{S}}_{i} \cdot \hat{\mathbf{S}}_{i+1}-(-)^{i} \mathbf{D} \cdot \hat{\mathbf{S}}_{i} \times \hat{\mathbf{S}}_{i+1}\right. \\
& \left.-\mu_{B} \mathbf{H} \cdot\left[\mathbf{g}^{u}+(-)^{i} \mathbf{g}^{s}\right] \cdot \hat{\mathbf{S}}_{i}\right)
\end{aligned}
$$

where the three terms in the summation are the antiferromagnetic Heisenberg, DM and Zeeman splitting interactions, respectively. The exchange coupling constant $J$, determined from the neutron scattering measurements, is about $1.57 \mathrm{meV}$. The DM interaction is much weaker than the Heisenberg term. The $\mathbf{D}$-vector, primarily aligned along the $a^{\prime \prime}$ axis, will be determined numerically. $\mathbf{g}^{u}$ and $\mathbf{g}^{s}$ are the uniform and staggered components of the alternating g-tensor as given in Ref. 13 for copper benzoate.

The DM term can in principle be eliminated by performing a spin rotation about the $\mathbf{D}$ vector by an angle $\alpha= \pm \frac{1}{2} \tan ^{-1}(D / J)$ on the alternating sites. This results in a small exchange anisotropy and an effective staggered magnetic field in addition to the $\mathbf{g}^{s}$-term in (11). The total effective staggered field is approximately given by

$$
\mathbf{H}_{s} \approx\left(\mathbf{g}^{s}-\frac{1}{2 J} \mathbf{D} \times \mathbf{g}^{u}\right) \cdot \mathbf{H}
$$

up to the leading order in $D / J$. In Ref. 9, OA studied the isotropic Heisenberg model with a staggered field after eliminating the DM term and neglecting all anisotropic terms. They estimated that the DM coupling constant is about $\mathbf{D}=(0.13,0.0,0.02) \mathrm{J}$ from the specific heat, neutron scattering and ESR measurement data [1, 9].

In order to explore the low-energy properties of copper benzoate, we use density matrix renormalization group (DMRG) 14, 15] to study directly the Hamiltonian defined by Eq. (1). Since neither the total spin nor its z-component is a good quantum number, the calculation 
is computer time consuming. Open boundary conditions are used and up to 400 states are retained in our calculation. The convergence of the results is systematically examined and the truncation error is less than $10^{-7}$. To obtain the values of gaps and magnetizations in the thermodynamic limit, an extrapolation from the DMRG calculations up to 1000 sites is done.

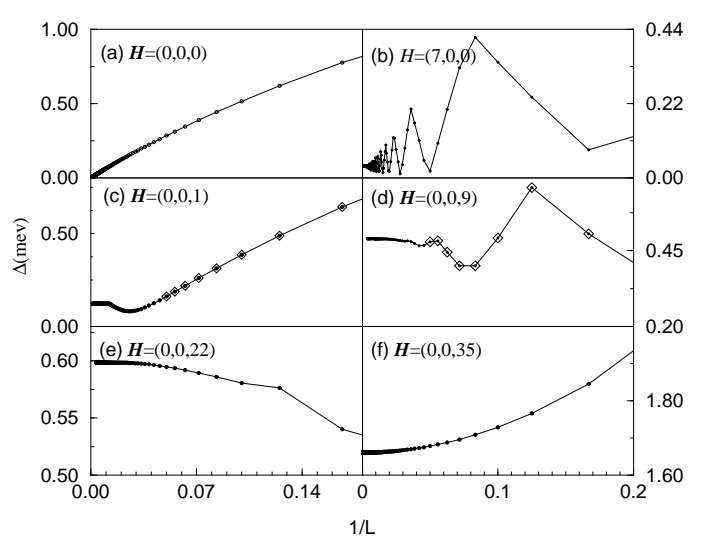

FIG. 1: Spin gaps versus 1/L in different applied fields for $\mathbf{D}=(0.13,0.0,0.02) J$. The exact diagonalization results $(\diamond)$ for small lattices are also shown for comparison.

Figure 1 shows the spin excitation gap as a function of inverse lattice size $L$ in different applied fields $H$. In zero field, there is no gap in the excitation spectrum in the thermodynamic limit and the gap decreases monotonically with $L$ increasing. However, in finite fields, the size dependence of the gap becomes more complicated and changes dramatically with $H$ increasing. The oscillations of the gaps are due to the competition between the uniform and staggered magnetic fields. The most pronounced oscillation occurs when the contributions to the Zeeman energy from these two fields become comparable, and the characteristic length scale of the oscillation is inversely proportional to the energy gap. Since the gap along $a^{\prime \prime}$-axis is much smaller than other directions, the oscillation looks stronger for $H=(7,0,0) T$ than for other cases.

Figure 2 shows the field dependence of the gap with $\mathbf{D}=(0.13,0.0,0.02) J$. In low fields, we find that the gap is well described by the following equation

$$
\Delta_{\alpha}=C^{\alpha} H^{2 / 3} \ln ^{1 / 6}\left(J / \mu_{B} H_{s}\right),
$$

as predicted by Oshikawa and Affleck. By fitting the numerical data with the above equation, we find that the coefficient $C^{\alpha}$ is given by $C=(0.0072,0.059,0.097)$ for $\mathbf{D}=(0.13,0.0,0.02) \mathrm{J}$. In this system, the gap is finite even in very low field. This is completely different from the pure Heisenberg model whose excitations remain gapless until the spin polarization induced by the applied field becomes saturated. This difference results from the effective staggered magnetic field induced by

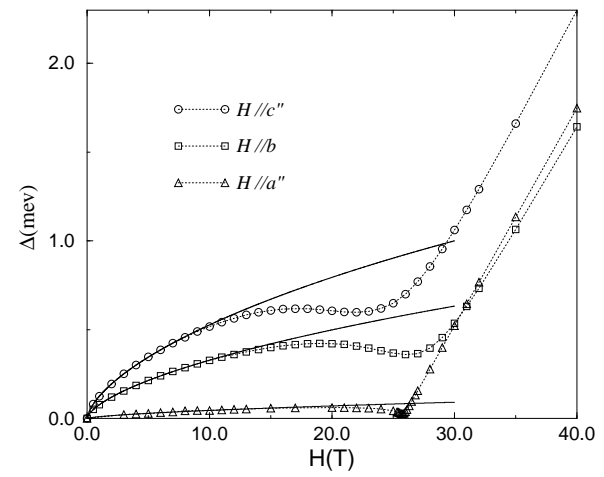

FIG. 2: Field dependence of the spin excitation gap. $\mathbf{D}=$ $(0.13,0.0,0.02) J$. The solid lines are the fitting curves of the numerical results with Eq. (3) the low fields.

the alternating g-tensor and the DM interaction. Since this staggered field couples directly to the strongest spin fluctuation mode at $q=\pi$, it gives rise to the instability of the ground state and opens a gap in the excitation spectrum. In high fields, the gap varies almost linearly with $H$ and its slope is proportional to the corresponding value $g_{\alpha \alpha}^{u}$. In the intermediate field regime, the gap varies non-monotonically with $H$ and shows a local minimum around $H \sim 25 T$ in all three directions. This non-monotonic behavior of the gap is also due to the competition between the applied field and the induced staggered field. However, we find that this competition does not lead to a phase transition in the ground state in this crossover regime, since the ground state energy drops continuously and smoothly with $H$.

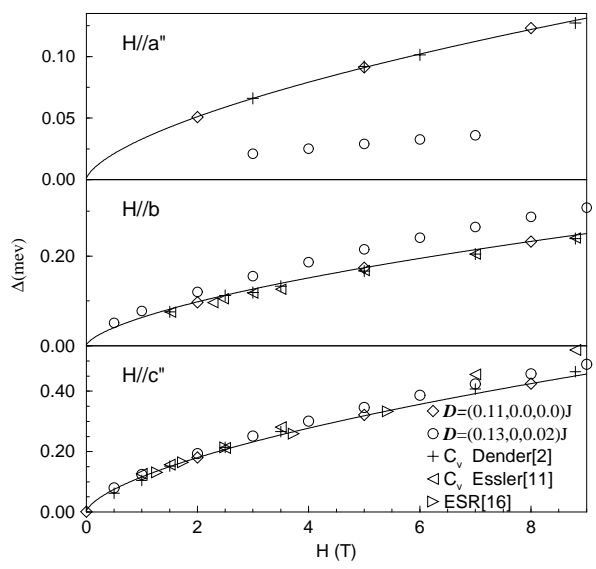

FIG. 3: Comparison between numerical results ( $\circ$ and $\diamond)$ and the specific heat $C_{v}(+, \triangleleft)$ and $\operatorname{ESR}(\triangleright)$ data for the field dependence of energy gaps. Solid lines are the fitting curves of the DMRG results to $\Delta_{\alpha}=C_{\mathrm{DMRG}}^{\alpha} H^{2 / 3} \ln ^{1 / 6}\left(J / \mu_{B} H_{s}\right)$ for $\mathbf{D}=(0.11,0.0,0.0) J$ with $C_{\mathrm{DMRG}}=(0.025,0.048,0.089)$.

Now let us turn to the issue whether the model (11) is sufficient to describe the low-energy magnetic properties of copper benzoate. In the effective field theory, the critical exponents of the gaps are determined 
by the relevant and marginal operators in the low field limit. However, as this theory contains only the contribution of the dominant effective staggered field and ignores all other anisotropic terms after eliminating the DM term, it cannot explain the field-dependence of the gaps along all three principal axes. Moreover, since the energy gap is very sensitive to the DM interaction, the value of $\mathbf{D}$-vector determined from this theory, $\mathbf{D}=(0.13,0.0,0.02) J[9]$, would also be inaccurate.

To determine accurately the value of $\mathbf{D}$, we have evaluated and analyzed the field dependence of the gaps around $\mathbf{D}=(0.13,0.0,0.02) \mathrm{J}$. By comparison with experiments [2], we find that the gaps for $\mathbf{D}=$ $(0.11,0.0,0.0) J$ give the best fit to the experimental data in all three directions (Figure 3). It also agrees with the recent ESR data for $H / / c^{\prime \prime} 16$ as well as the results of Essler for $H / / b$ and in low fields for $H / / c^{\prime \prime}[11]$. For this D value, our numerical results for the gap ratios $\Delta_{a^{\prime \prime}}$ : $\Delta_{b}: \Delta_{c^{\prime \prime}}$ in low fields are $1: 1.92: 3.56$, in agreement with experiments. However, for $\mathbf{D}=(0.13,0.0,0.02) \mathrm{J}$, the low field gap ratios are $1: 8.2: 13.5$ and the theoretical results agree with the experimental data only along the $c^{\prime \prime}$-axis (Figure 3). Therefore the Hamiltonian (1) is indeed an appropriate model for copper benzoate and the discrepancy between the effective field theory and experiments is due to the neglect of the anisotropic terms rather than due to the interchain coupling as previously suggested [9].

For the $\mathrm{S}=1 / 2$ Heisenberg model, the momentum of the antiferromagnetic soft mode is shifted from $\pi$ to $\pi \pm \delta q$ in an applied field. This leads to an incommensurate peak in the longitudinal structure factor. The momentum shift is predicted to be proportional to the magnetization:

$$
\delta q=2 \pi M(H) .
$$

This linear relationship between the momentum shift and the magnetization was examined by Dender at al for copper benzoate 2]. They found that the momentum shifts at $H=3.5,5,7 T$ for $\mathbf{H} / / b$ were consistent with the theoretical results for the pure Heisenberg model [17] with $g=2.059$ and $J=1.57 \mathrm{meV}$. However, as the excitation spectrum of copper benzoate is fully gaped even in an arbitrarily small field, unlike in the pure Heisenberg model, this issue needs also to be reexamined. To do this, we have calculated the spin-spin correlation functions

$$
S_{q}^{\alpha \alpha}=\frac{1}{N} \sum_{i j}\left\langle\left(S_{i}^{\alpha}-\left\langle S_{i}^{\alpha}\right\rangle\right)\left(S_{j}^{\alpha}-\left\langle S_{j}^{\alpha}\right\rangle\right)\right\rangle e^{i q\left(R_{i}-R_{j}\right)} .
$$

The momentum shift $\delta q$ can be determined from the peak position of $S_{q}^{\alpha \alpha}$. From our calculations, we find that $S_{q}^{\alpha \alpha}$ behaves differently along the three principal axes. $S_{q}^{b b}$ shows a weak incommensurate peak. However the peaks of $S_{q}^{a^{\prime \prime} a^{\prime \prime}}$ and $S_{q}^{c^{\prime \prime} c^{\prime \prime}}$ are pinned at $q=\pi$. With $H$ increasing, the heights of these peaks are suppressed in all three directions.

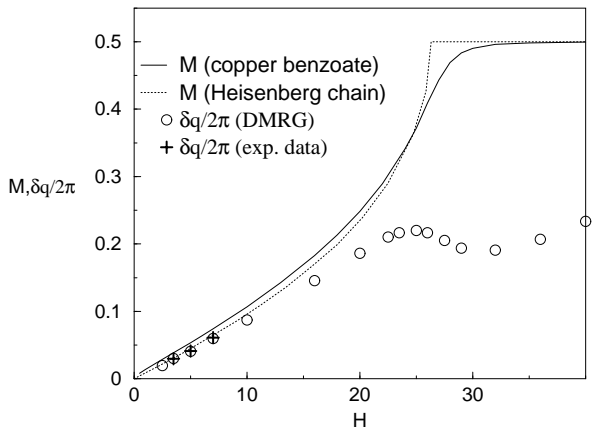

FIG. 4: Magnetization $M$ versus the applied field $H$ with $\mathbf{D}=(0.11,0,0) J$ (dashed line) and the pure Heisenberg model (solid line) for $H / / b$. The corresponding shift $\delta q / 2 \pi$ (o) is shown and also compared with the experimental data $(+)$ [2].

Figure (44) shows the field dependence of the magnetization $M(H)$ and the momentum shift $\delta q$ for $\mathbf{H} / / b$. For comparison, the magnetization curve for the pure Heisenberg model and the experimental data for $\delta q$ are also shown in the figure. As seen from the figure, our numerical values of $\delta q$ agree quantitatively with the experimental data 2]. However, we find that Eq. (44) is not valid for copper benzoate. In particular, in contrast to $M(H), \delta q$ varies non-monotonically with $H$. We believe this non-monotonic variation of $\delta q$ with $H$ can be detected by neutron scattering measurements. In addition, the magnetization is gradually saturated for large fields as a direct consequence of the DM interaction. This is different from in the pure Heisenberg model where the spins are fully polarized beyond a critical field $\mu_{B} H_{c}=J$, at which the two magnetization curves differ mostly.

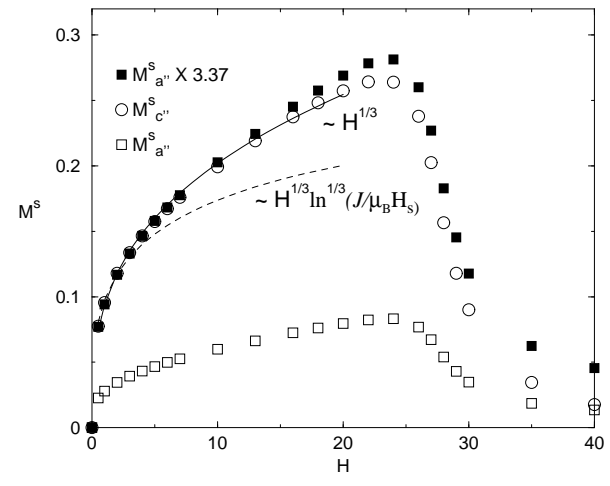

FIG. 5: The staggered magnetization as a function of $H$ for $\mathbf{H} / / b$ and $\mathbf{D}=(0.11,0,0) J$

When $\mathbf{H} / / b$, spins are polarized ferromagnetically along the $b$-axis and antiferromagnetically along the other two directions. Figure (5) shows the staggered magnetization as a function of $H$ for $\mathbf{D}=(0.11,0,0) \mathrm{J}$. At low fields, both $M_{a^{\prime \prime}}^{s}$ and $M_{c^{\prime \prime}}^{s}$ behave as $H^{1 / 3}$. This field dependence of the staggered magnetization above $2 T$ deviates from the $H^{1 / 3} \ln ^{1 / 3}\left(J / \mu_{B} H_{s}\right)$ behavior pre- 
dicted by the effective field theory [9] and is not sensitive to the $\mathbf{D}$-vector. It may well be that the range of the validity of the leading approximation in the effective field theory is narrower for the staggered magnetization than that for the gap. We should also mention that the numerical accuracy is lower when $H$ is below $0.5 \mathrm{~T}$. In intermediate fields, both $M_{a^{\prime \prime}}^{s}$ and $M_{c^{\prime \prime}}^{s}$ vary non-monotonically with $H$, in analogy with the field dependence of energy gaps. In the large $H$ limit, $M_{s}$ tends to approach zero. This means that the staggered field $H_{s}$ induced by the external field is overwhelmed by the uniform field $H$ in the high field limit, although $H_{s}$ is approximately proportional to $H$.

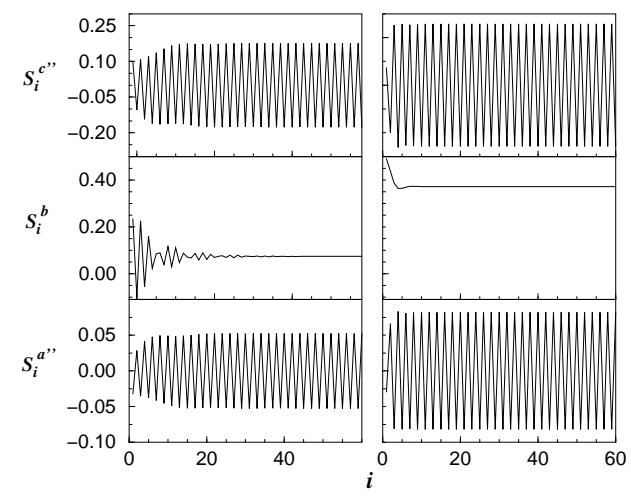

FIG. 6: Local spin polarization for $\mathbf{H}=(0,7,0) T$ (left) and $\mathbf{H}=(0,25,0) T$ (right) for $L=120$ and $\mathbf{D}=(0.11,0,0) J . S_{i}^{b}$ is symmetric with respect to the middle point of chain, while the others are antisymmetric. Only is half of the chain shown.

The above results show that the DM interaction affects strongly the properties of low-lying excitations although this term is much smaller than the Heisenberg exchange interaction. In particular, the staggered magnetic field induced by this term opens a gap in the spin excitations and leads to an antiferromagnetic long range order to accompany the ferromagnetic long range order induced by the applied field, shown in Fig 6. The coexistence of orthogonal ferromagnetic and antiferromagnetic long range orders in an external field is a characteristic feature of quantum spin systems with DM interactions.

In conclusion, we have shown that the microscopic model (1) including two inequivalent lattice sites and the DM interaction is a very good description of available experimental data. It can reproduce correctly the gap values in all three directions as well as the shift of the incommensurate peaks, without resorting to any additional interaction like interchain coupling. The crossover to strong field behavior and the breakdown of the rela- tion between the momentum shift and the magnetization call for further experimental studies. Finally, we would point out that although the DM interaction is generally small, its effects could be very important for real materials. In particular, for metallic materials, e.g. $\mathrm{Yb}_{4} \mathrm{As}_{3}[3]$, one could use external fields to modulate the magnetic transport properties which is one of the focal points for the spintronics.

We wish to thank D.C. Dender and C. Broholm for correspondence and I. Affleck, C. F. Chen, F. Essler, H. Nojiri, M. Oshikawa and K. Ueda for fruitful discussions. This work is supported in part by the Special Funds for Major State Basic Research Projects of China and by the National Natural Science Foundation of China.

[1] D.C. Dender, D. Davidovic, D.H. Reich and C. Broholm and G. Aeppli, Phys. Rev. B 532583 (1996).

[2] D.C. Dender, P.R. Hammar, D.H. Reich and C. Broholm and G. Aeppli, Phys. Rev. Lett. 791750 (1997).

[3] M. Kohgi, K. Iwasa, J. Mignot, B. Fak, P. Gegenwart, M. Lang, A. Ochiai, H. Aoki, and T. Suzuki, Phys. Rev. Lett. 86, 2439(2001).

[4] P. Fulde, B. Schmidt, and P. Thalmeier, Europhys. Lett. 31, 323(1995).

[5] M. Oshikawa, K. Ueda, H. Aoki, A. Ochiai and M. Kohgi, J. Phys. Soc. Jpn, 68, 3181(1999); H. Shiba, K. Udea, and O. Sakai, J. Phys. Soc. Jpn, 69, 1493(2000).

[6] I. Tsukada, J. Takeya, T. Masuda and K. Uchinokura, Phys. Rev. Lett. 87, 127203(2001).

[7] I. Dzyaloshinskii, J. Phys. Chem. Solids 4, 241(1958)

[8] T. Moriya, Phys. Rev. 120, 91 (1960)

[9] M. Oshikawa and I. Affleck, Phys. Rev. Lett. 79, 2883(1997); I. Affleck and M. Oshikawa, Phys. Rev. B60, 1038(1999).

[10] A. Fledderjohann, C. Gerhardt, K.H. Mütter, A. Schmitt, and M. Karbach, Phys. Rev. B54, 7168(1996).

[11] Fabian H.L. Essler, Phys. Rev. B59, 14376(1999).

[12] J.Z. Lou, C.F. Chen, S.J. Qin, Z.B. Su and L. Yu, Phys. Rev. B 65, 64420 (2002)

[13] M. Date, M. Mutokawa, and. H. Tamazaki, J. Phys. Soc. Jpn. 18, 911(1963); K. Oshima, K. Okuda and M. Date J. Phys. Soc. Jpn. 41, 475(1976).

[14] S.R. White, Phys. Rev. Lett. 69, 2863(1992).

[15] I. Peschel, X. Wang, M. Kaulke and K. Hallberg, Density Matrix Renormalization, LNP, 528, (Springer-Verlag, New York, 1999).

[16] T. Asano, H. Nojiri, Y. Inagaki, J. P. Boucher, T. Sakon, Y. Ajiro and M. Motokawa, Phys. Rev. Lett. 84 5880(2000). H. Nojiri, private communication.

[17] G. Müller, H. Thomas, H. Beck, J.C. Bonner, Phys. Rev. B24, 1429 (1981). 